\title{
O Design Participativo como oportunidade para a implementação da Gestão do Conhecimento em PMEs
}

\author{
Amanda Carolina Teixeira ${ }^{1}$, Nelson Nunes Tenório Junior ${ }^{2}$, Arthur Gualberto \\ Bacelar da Cruz Urpia ${ }^{3}$ \\ ${ }^{1}$ Mestranda em Gestão de Conhecimento nas Organizações pelo Centro Universitário de \\ Maringá (Unicesumar) - Maringá, PR - Brazil \\ ${ }^{2}$ Professor do Centro Universitário de Maringá (Unicesumar) - Maringá, PR - Brazil \\ ${ }^{3}$ Professor do Centro Universitário de Maringá (Unicesumar) - Maringá, PR - Brazil \\ manditex@gmail.com, nelson.tenoriojr@gmail.com, arthur.urpia@unicesumar.edu.br
}

\begin{abstract}
The Small and Medium Enterprises (SMEs) are the main source of jobs and local economic development. Nonetheless, in the current context in which economies are based on knowledge, they are not used to implement Knowledge Management (KM) deliberately. As a result, the aim of this research is to investigate which oportunities Participatory Design can bring to the process of KM implementation in SMEs, in light of the three main critical successful factors found in the literature. For this purpose, a etnografic study has been carried out in a working group called 'Squad of KM' of a medium-sized consultancy company, located in São Paulo, whose goal is to implement KM in the whole enterprise. The results show the existence of various oportunities presented by PD for KM in SMEs based on the literature and the working group's activities.
\end{abstract}

Resumo. As Pequenas e Médias Empresas (PMEs) são as principais fontes de criação de empregos e de desenvolvimento econômico local. Apesar disso, no contexto atual em que as economias são baseadas em conhecimento, elas não costumam praticar a Gestão do Conhecimento $(G C)$ de forma deliberada. Com efeito, o objetivo desta pesquisa é investigar quais oportunidades o Design Participativo (DP) pode trazer ao processo de implementação da GC em PMEs, à luz dos três principais fatores críticos de sucesso encontrados na literatura. Para tanto, foi conduzida uma etnografia em um grupo de trabalho (Squad de GC) de uma média empresa de consultoria, localizada em São Paulo, cujo escopo é implementar a GC na companhia. Os resultados mostram a existência diversas oportunidades apresentadas pelo DP para a implementação da GC em PMEs baseado nos achados da literatura e de elementos de DP nas atividades do grupo de trabalho.

\section{Contextualização do problema em investigação}

Este artigo tem por enfoque investigar quais as oportunidades apresentadas pelo Design Participativo (DP) no processo de implementação da Gestão do Conhecimento (GC) em Pequenas e Médias Empresas (PMEs). No contexto atual em que as economias são cada vez mais baseadas em conhecimento, verifica-se que as PMEs são as principais fontes de criação de empregos e de desenvolvimento econômico local. Apesar disso, são empresas dotadas de escassos recursos e precisam desenvolver capacidades dinâmicas para manterem-se competitivas em um mercado turbulento e em constante mudança [North e Babakhanlou, 2016]. As capacidades dinâmicas envolvem a habilidade de uma 
empresa para integrar, construir e reconfigurar competências internas e externas para gerir ambientes em constante mudança, o que pode envolver ajustamentos das rotinas operacionais e dos próprios recursos produtivos de um modo considerado apropriado pelos seus principais tomadores de decisões [Teece et al., 1997; Cepeda-Carrion et al., 2017].

Nesse contexto, a GC, que se preocupa com a exploração e desenvolvimento dos ativos de conhecimento de uma organização com vista a promover seus objetivos [Davenport e Prusak, 2005], torna-se um componente importante para o desenvolvimento das capacidades dinâmicas e o aprendizado organizacional, conforme Pavlou e El Sawy (2011). Contudo, verificam-se alguns entraves para a implementação da GC em PMEs, chamados fatores críticos de sucesso, como a ausência de uma estratégia clara para a GC, a falta de uma cultura de compartilhamento, a falta de apoio da liderança e de comprometimento dos gestores [Wong e Aspinwall, 2005; Miklosik e Zak, 2015]. Considerando a importância das PMEs do ponto de vista da estratégia e da inovação, são necessárias estratégias que promovam a transferência de conhecimento e experiência no seio das organizações [Durst e Edvardsson, 2012].

Como forma de oportunizar a implementação da GC nessas empresas, tendo por ponto de partida os fatores críticos de sucesso, vislumbra-se a relevância do DP. Cada vez mais aplicado nos projetos de design, tem como uma de suas características a de proporcionar a participação dos usuários para, por meio da empatia e da comunicação, compreender seus comportamentos e o que, de fato, eles desejam durante sua experiência com o produto, possibilitando a criação de novos modos atrativos para a experiência do usuário [Mahlamäki, 2013; Wang et al., 2017]. É intensamente colaborativo, envolve conhecimentos multidisciplinares e lida com problemas complexos, cuja solução demanda uma compreensão mais profunda de sua natureza [Bratteteig e Wagner, 2014].

Tendo essas ideias por base, este estudo busca identificar as oportunidades que o DP apresenta para entabular a implementação da GC em PMEs, pois possibilita a colaboração e a criação de interações e conexões entre os participantes para a concepção de iniciativas de GC atacando, por conseguinte, os principais fatores críticos de sucesso. Para se alcançar o objetivo desta pesquisa, analisou-se o contexto da GC em PMEs e seus fatores críticos de sucesso. Em seguida, procedeu-se à uma revisão de literatura para investigar a utilização do DP no campo da GC. Por fim, analisou-se o processo de implementação da GC em uma PME, por meio da etnografia, a fim de se identificar quais oportunidades o Design Participativo pode apresentar a esses contextos.

\section{A GC em PMEs}

A GC tem sido reconhecida por auxiliar na performance organizacional por meio da implementação de ferramentas, processos, sistemas, estruturas e culturas para melhorar os processos de criação, compartilhamento e uso de conhecimentos, cruciais para a inovação, a competitividade e a tomada de decisão das empresas [Despres e Chauvel, 2001]. No sistema econômico atual, o conhecimento é um dos principais recursos das organizações, sendo a sua gestão uma das principais dificuldades das PMEs. Elas concentram a maior parte do conhecimento, predominantemente tácito, nos sócios e 
gestores, possuem raríssimo conhecimento registrado e não o armazenam adequadamente para posterior recuperação e o uso [Durst e Edvardsson, 2012]. Por isso, o desafio central da GC é, em nível pessoal e organizacional, facilitar aqueles processos para a vantagem da organização e dos consumidores, a exemplo de quando um conhecimento de excelência já existente é aplicado na produção de um produto de alta tecnologia ou na criação de designs criativos que tornem os produtos fáceis de usar, sejam de baixo custo e úteis ao consumidor [Wiig, 1993].

As PMEs são os tipos mais comuns de empresas ao redor do mundo [Durst e Bruns, 2018], chegando a representar 90\% dos negócios mundiais, 54\% do total de empregos formais e $20 \%$ do Produto Interno Bruto (PIB) do Brasil [Sebrae, 2018]. Sua importância nas economias modernas é irrefutável, pois muitas operam com elevados níveis de eficiência e dinamismo, devido a sua capacidade de adaptação e resposta [Yu, 2001; Massaro et al., 2016]. O sucesso das PMEs brasileiras de crescimento acelerado dependerá cada vez mais de inovação, eficiência e investimento em capital humano, buscando disseminar uma cultura aberta a novas soluções e investir em Pesquisa e Desenvolvimento [Deloitte, 2019], o que reforça o papel da GC nesses contextos.

Dentre as principais características que distinguem as PMEs, destaca-se a responsabilidade pessoal do proprietário pelas atividades da empresa, o qual tende a concentrar os processos de planejamento do negócio e tomada de decisão. Outra característica é a produção de produtos e serviços customizados e a facilidade de adaptação a mudanças no ambiente de negócios [Durst e Bruns, 2018]. A maioria das PMEs não possui uma estratégia de GC e nem demostram a necessidade de processos formalizados, tratando o tema em nível operacional, com ênfase no gerenciamento do conhecimento tácito [Durst e Edvardsson, 2012; Wee e Chua, 2013]. No entanto, seus benefícios são inegáveis: crescimento nas vendas, desenvolvimento dos empregados (habilidades e aprendizado), satisfação do consumidor (fidelização), inovação, criatividade, melhor relacionamento com outras firmas, melhorias na gestão de recursos humanos o desempenho organizacional [Durst e Bruns, 2018]. Apesar de sua importância, as PMEs nunca foram objeto de muita atenção no campo da GC, pois avanços na área tradicionalmente visaram às grandes organizações. Nas duas últimas décadas, no entanto, o tema vem ganhando importância nos círculos acadêmicos, práticos e de políticas públicas [Cerchione et al., 2016; Mishra, 2019].

Alguns processos de GC são facilitados pelas características das PMEs, como o compartilhamento do conhecimento, que é favorecido pelo ambiente de familiaridade, confiança e interação social, proximidade, elevada frequência de comunicação, facilidade na disseminação de informações, entrecruzamento de funções e sobreposição de atividades entre os colegas. Já o processo de armazenamento e retenção de conhecimento são os principais desafios para as PMEs, uma vez que as soluções são customizadas, diferenciadas e o processo de armazenamento demanda recursos financeiros e tempo para formalidades em sistemas e procedimentos, de modo que o conhecimento acaba se concentrado na mente dos proprietários e gestores [Wong e Aspinwall, 2004; Durst e Edvardsson, 2012; Wee e Chua, 2013].

Para o florescer de uma iniciativa de GC, portanto, são necessários alguns fatores críticos de sucesso: atividades e práticas que devem ser direcionadas para se garantir a implementação da GC [Wong, 2005]. Tendo em vista as dificuldades de projetos de GC, 
como a falta de compreensão de seus inibidores, a compreensão desses fatores pode auxiliar as firmas em sua avaliação, planejamento e implementação [Bahrami e Oktar, 2017]. Wong e Aspinwall (2005) elencam 11 fatores críticos de sucesso para a implementação da GC em PMEs, nesta ordem de importância: apoio da alta direção e liderança, cultura que favoreça o compartilhamento, estratégia clara e propósito para a GC, recursos, processos e atividades, treinamento e educação, gestão de recursos humanos, tecnologia da informação, auxílio motivacional, infraestrutura organizacional e avaliação. A importância dos três primeiros aspectos também foram destacados em outros estudos [Yang et al., 2010; Chang e Wang, 2009; Anand et al., 2012; Miklosik e Zak, 2015].

Desse modo, embora as ferramentas de GC auxiliem no processo de gerar, codificar e transferir conhecimento dentro e fora das organizações [Ruggles, 1998], sua efetividade depende da existência de uma cultura de colaboração e de compartilhamento do conhecimento [Servin, 2005]. Davenport e Prusak (1998) e Delong e Fahey (2000) afirmam que aspectos relacionados às pessoas sejam muito superiores ao da tecnologia no apoio à GC, conforme se verfica dos próprios fatores supracitados. O critério 'pessoas' é reforçado quando O'Dell e Grayson (1998), Dorow (2017) e Govella (2019) destacam a confiança como primordial na formação dessa cultura de compartilhamento do conhecimento, favorecendo a disposição das pessoas em colaborarem. Nesse contexto, o uso do DP no processo de implementação da GC mostra-se com potencial para reduzir as chances de insucesso de uma iniciativa de GC em PMEs, na medida em que estimula o envolvimento dos empregados no design e avaliação de seu próprio trabalho, além da integração com pessoas de diversas áreas para criar novos conhecimentos e resolver problemas complexos.

\section{Design Participativo como oportunidade para o processo de implementação da GC em PMEs}

O DP vem sendo cada vez mais aplicado nos projetos de design, especialmente na área da computação, saúde e planejamento urbano. Dentre suas várias aplicações, proporciona a participação dos usuários no processo de design para que, por meio da empatia e da comunicação, os designers compreendam o comportamento dos usuários, suas necessidades e desejos durante a experiência com o produto, possibilitando a criação de contextos favoráveis à melhor experiência de usuário [Mahlamäki, 2013; Wang et al., 2017]. O aspecto fundamental do DP é que as pessoas deixam de serem rotuladas como usuários, consumidores ou clientes para serem participantes do processo de criação devido à crescente necessidade de inovação imposta às empresas que concorrem em mercados cada vez mais dinâmicos, colocando o design a níveis estratégicos nas organizações [Straioto e Figueiredo, 2015].

O DP envolve diferentes atores dentro das atividades do processo de design, envolvendo designers, equipes de desenvolvimento e parceiros externos (como fornecedores e os próprios consumidores), com diferentes papeis, experiências e contextos dentro do projeto [Chen et al., 2018]. Eles auxiliam toda a empresa a criar melhores experiências ao usuário com esses produtos [Govella, 2019], tornando-o mais aceito, acessível e usável, pois enfatiza a atuação dos usuários no decorrer do processo de desenvolvimento, levando em conta sua maneira de pensar e agir [Camargo e Fazani, 
2014; Chen et al., 2018]. O processo de cocriação do DP permite que os beneficiários do projeto tornem-se parceiros e membros da equipe de desenvolvimento em todas as fases, não servindo apenas de fontes de informação para as ideias dos designers [Scariot et al., 2012; Bratteteig e Wagner, 2014]. Nesse sentido, abordagens participativas enfatizam as diferenças de percepção entre os atores e valoriza, inclusive, a falta de consenso. Com efeito, o diálogo e a comunicação são priorizados para se chegar à solução buscada dentro do processo coletivo de aprendizado [Berthet, 2016].

Dentro do campo da GC, o processo de compartilhamento do conhecimento envolve possibilitar que uma pessoa acompanhe o pensamento de outrem, envolvendo processos de aprendizagem prolongados, que ocorrem a partir das trocas de experiências de forma voluntária, dentro de um contexto de participação em grupo [Dorow, 2017]. Hoffmann e Herrmann (1999) destacam que a participação cria maior motivação, pois as pessoas têm a oportunidade de influenciar o design de um sistema, possibilitando que suas ideias e sugestões sejam implementadas, de modo a alcançar o melhor sistema de acordo com os interesses das pessoas da organização. Logo, há um sentido em incluir os beneficiários da GC no processo de sua concepção e implementação, na medida em que a aprendizagem e a interação geradas têm o potencial de proporcionar efeitos positivos em atividades de compartilhamento de conhecimentos futuros dentro do âmbito organizacional.

Em revisão de literatura sobre o tema, os autores identificaram pesquisas que relacionam DP e GC [Valtolina et al. 2012; van Der Bijl-Brouwer e van Der Voort, 2014; Berthet et al., 2016; Wang et al., 2017 Sakellariou et al., 2017]. Esses autores destacam ser essencial para a GC a colaboração dos participantes no compartilhamento e interação com diferentes tipos de conhecimentos e o aprendizado coletivo, seja pela mediação tecnológica ou ferramentas como storytelling [Sakellariou et al., 2017] ou workshops de criação [van Der Bijl-Brouwer e van Der Voort, 2014]. Parte-se do pressuposto de que o uso do DP com o envolvimento de diversos profissionais e níveis hierárquicos pode favorecer o processo de implementação da GC nas empresas, analogamente ao design de sistemas de computador, por exemplo.

\section{Procedimentos metodológicos}

Por meio de um estudo qualitativo e exploratório, buscou-se compreender quais oportunidades o DP apresenta à implementação da GC, por meio de uma pesquisa etnográfica - com a observação participante e pesquisa documental como técnica de coleta de dados, de um grupo de trabalho, denominado Squad de GC da X-fin (nome fictício), uma PME brasileira do ramo de consultoria, com 28 anos no mercado e 60 membros, voltada para soluções de Tecnologia da Informação para gestão financeira de grandes empresas. No planejamento estratégico da X-Fin, designaram-se comitês estratégicos multidisciplinares e colaborativos formados por sócios e colaboradores denominados Squads (modelo que separa os funcionários em pequenos grupos multidisciplinares em prol de executarem objetivos específicos). No total, a empresa criou sete comitês: Responsabilidade Social, Capacitação, Liderança, Rentabilidade, Eficiência Comercial, Qualidade e Gestão do Conhecimento.

Neste trabalho, a análise recaiu sobre o comitê de GC, criado em março de 2020, responsável pela implementação da GC na organização, formado por oito membros, 
sendo seis membros de áreas distintas da empresa, uma consultora da área de treinamento, que tem o encargo de facilitar as reuniões com metodologias próprias para se alcançar os objetivos do grupo, além de uma pesquisadora da área de GC responsável pela condução da etnografia, que integrou o grupo no mês de junho de 2020. O objetivo do Squad é estruturar práticas de GC que padronizem, facilitem e aumentem a velocidade da troca de conhecimentos dentro da organização, acelerando a curva de aprendizado dos colaboradores, contribuindo com conhecimentos e habilidades específicas de sua atuação que não são encontradas em treinamentos externos.

Destacam-se as seguintes etapas percorridas pelo grupo: a) o mapeamento dos conhecimentos críticos da organização por meio da ferramenta Design Thinking em cada uma das quatro unidades de negócio pela consultora envolvendo $30 \%$ dos colaboradores da organização; b) a identificação de práticas de GC adequadas aos conhecimentos mapeados; c) a seleção de duas práticas já existentes na companhia para serem estruturadas no projeto piloto: 1) um evento de compartilhamento - palestras conduzidas por membros da empresa sobre temas específicos de alguma unidade de negócio e 2) produção de artigos técnicos com dicas rápidas para resolver algum problema.

A empresa foi selecionada pelo critério de conveniência e acessibilidade, pois tinha as características ideais para o desenvolvimento da pesquisa. Neste caso, a pesquisadora participante das reuniões semanais de alinhamento das atividades do Squad de GC ao longo do período de cinco meses - 30 de junho a 01 de dezembro de 2020, atuou com o intuito de identificar quais oportunidades o DP apresenta para a implementação da GC em PMEs. Foram feitos diários de campo de um total 22 reuniões de $2 \mathrm{~h}, 100 \%$ remotas, via plataforma Teams, ocorridas no contexto da pandemia do coronavírus. Ressalta-se que houve consentimento informado da empresa e dos participantes, após esclarecidos os objetivos da pesquisa, e a pesquisadora foi bem recebida e acolhida com naturalidade pelos membros do grupo, interessados em saber mais sobre a GC. Além disso, faz parte da cultura da empresa convidar membros externos detentores de conhecimentos específicos para contribuir em projetos.

A etnografia permite flexibilidade para a obtenção de informações, o que é importante para a análise de contextos culturais com os quais o pesquisador não está muito familiarizado [Leedy e Ormrod, 2016]. Buscando responder à pergunta de pesquisa analisou-se as "normas, crenças, estruturas sociais e outros padrões" do grupo, [Leedy e Ormrod, 2016, p.274]. Considerando-se que um dos fatores críticos que se vislumbra analisar neste trabalho é a cultura organizacional, destaca-se a importância da etnografia, pois se pode experimentar o contexto cultural e sua compreensão, facilitando a interpretação dos dados [Kozinets, 2014], que seriam quase impossíveis de se obter e compreender utilizando outra abordagem. Quando o pesquisador se engaja em uma observação participante como um integrante do grupo, há uma vantagem para a obtenção de informações [Leedy e Ormrod, 2016], pois se mergulha nas maravilhas dos contextos combinando a observação, a audição cuidadosa, e a participação em eventos da comunidade [Silverman, 2009; Schuler e Namioka, 2015].

O pesquisador responsável pela observação participante procedeu à anotações de campo de cada uma das reuniões, análise das atas das respectivas reuniões, bem como de outros documentos, apresentações de slides, artefatos e registros do grupo para captar perspectivas pessoais, processos sociais do grupo e da organização como um todo, dos 
arranjos hierárquicos, das estratégias da organização e o modelo mental de seus membros, rituais e comportamentos consagrados na organização, conforme recomenda Kozinets (2014). Foram registradas em um diário de campo: a) os objetivos de cada reunião, b) as opiniões divergentes e convergentes, c) as decisões principais de cada reunião, e) o ponto de vista dos participantes sobre cada tema.

De posse de todas esses registros, procedeu-se à leitura desses diários e das atas de reuniões confeccionadas pela consultora facilitadora das reuniões (parte documental), pontuando elementos relacionados ao DP. Após a leitura de todos esses materiais, constatou-se, indutivamente, que os temas dessas reuniões guardavam forte relação com os fatores críticos de sucesso estudados. Com efeito, numa segunda leitura desses diários e notas de campo, passou-se à codificação dos principais temas das reuniões, conforme sua relação com os fatores críticos considerados mais relevantes por Wong e Aspinwall (2005): "estratégia clara para a GC", "liderança" e "cultura organizacional", numa análise categorial de conteúdo, identificando "núcleos de sentido" [Bardin, 1997] dos contextos observados, verificando valores, crenças e motivações. Nesse sentido, a realidade subjetiva também foi objeto de estudo nesse processo de interpretação. Por fim, identificou-se os elementos de DP em cada fator crítico analisado por meio de inferência e interpretação, vislumbrando o modo como esses podem oportunizar o processo de implementação da GC em PMEs.

\section{Apresentação e análise dos dados}

Os temas analisados no Squad de GC da X-fin foram classificados de acordo com as categorias relativas aos três principais fatores críticos de sucesso citados pela literatura "estratégia clara para a GC", "liderança" e "cultura organizacional", identificando neles elementos de DP que apresentem oportunidades ao processo de implementação da Gestão do Conhecimento em PMEs.

\subsection{Estratégia Clara para a GC}

Em primeiro lugar, a definição da estratégia para a GC não foi idealizada com a participação ativa de toda a alta direção, apenas um sócio participou do Squad de GC. A despeito do acompanhamento das atividades do Squad pelo Conselho de Gestão Estratégica (CGE) da companhia, formado pelos sócios da companhia, com reuniões de prestação de contas mensais, verificou-se que o baixo nível de proximidade de outros membros da direção com as atividades do grupo tornou mais lento o entendimento dos sócios acerca do modo de funcionamento das iniciativas de GC, seus desafios e noções sobre o tempo do projeto. Outra inquietação dos sócios foi a percepção da necessidade de envolvimento Squad com outras iniciativas simultâneas e independentes de GC oriundas de outros Squads.

Apesar de não incluir toda a companhia, o grupo teve a preocupação com a definição de um objetivo para a GC. Para isso, utilizaram-se de uma técnica de visualização em grupo para a GC dentro da organização no ano de 2030 (um desdobramento da visão 2030 já existente na organização, que integra o planejamento estratégico da empresa). No processo de criação desse material, privilegiou-se a participação, pois, após a visualização individual, houve o compartilhamento das visões em pequenos grupos e posterior reescritura de um único documento ao final contendo as 
diversas opiniões. Uma oportunidade para a definição da estratégia teria a condução dessa dinâmica com uma metodologia de DP com a participação de todos os integrantes da empresa, a fim de que houvesse comprometimento dos membros da organização com o projeto conduzido pelo Squad de GC.

A opção do grupo pelo benchmark junto a outras companhias e consultorias para identificar práticas viáveis dentro do contexto da empresa também revelou a importância do DP no delineamento de estratégias de GC. Isso se verificou pela busca de conhecimentos multidisciplinares e de diversas fontes, a integração e participação do grupo em eventos e congressos com membros de outras empresas, a construção de um mapa mental colaborativo com os resultados dessas pesquisas, bem como estudos e discussões em conjunto.

A utilização da técnica de Design Thinking em algumas sessões para mapear conhecimentos relevantes à organização com a participação de $30 \%$ dos colaboradores também ressaltou a relevância do DP no processo de implementação da GC, pois foi possível identificar a necessidade de práticas voltadas para apoiar soluções de negócio e conhecimentos de difícil recuperação. Apesar de sua importância, nas atas das reuniões do Squad de GC relatou-se a inibição de alguns participantes das sessões o que dificultava a integração e a captura de informações importantes sobre os conhecimentos na ótica de todos os participantes.

Outro ponto estratégico relacionado com o DP foi a necessidade de integração com outros Squads da empresa, para apoiar práticas de GC que estão sendo estimuladas de forma independente nesses grupos, a exemplo do Squad de Qualidade, que busca aprimorar e inovar as metodologias de projetos. Desse modo, constata-se o potencial e importância da participação das pessoas no processo de implementação de uma iniciativa de GC pelo ponto de vista da estratégia.

\subsection{Liderança}

A Liderança da companhia é bem demarcada, conforme análise dos diários de campo das reuniões do Squad. Exemplar disso, é a reunião mensal dos oito sócios no CGE para debater temas estratégicos. Um dos problemas identificados nessa categoria foi a indisponibilidade de tempo do sócio para participar de forma mais intensa no treinamento dos consultores, que vão aprendendo com a prática e conforme surgem dúvidas. O Squad percebeu a necessidade da organização de que os conhecimentos dos sócios permaneçam mesmo quando esses não estejam mais presentes no negócio da empresa, o que reforça a importância da liderança para fomentar a integração com seus liderados em discussões estratégicas, em dar palestras e estimular consultores seniors no compartilhamento de conhecimentos e no processo de aprendizado de seus liderados para uma aprendizagem mais rápida.

Por outro lado, um ponto positivo é a participação de colaboradores não sócios para absorver as percepções dos sócios e auxiliar na correção de rumos das atividades dos Squads nas reuniões estratégicas dos sócios. A proposta do DP vislumbra justamente essa diversidade de percepções, sendo isso fundamental para uma iniciativa de GC. Além disso, o apoio dos líderes em uma iniciativa de GC emergente ao Squad de Qualidade, qual seja Hackathons trimestrais para fomentar o alcance de soluções para problemas do dia a dia, demonstra o quanto esse fator crítico de sucesso é essencial para 
engajar os membros da companhia a participar no processo criativo e inovativo. Um Hackathon tem claros elementos de DP presentes, pois integra pessoas com diversos níveis hierárquicos e de áreas diferentes para a solução de problemas complexos. Essa iniciativa teve impacto no Squad de GC, pois daí surgiu a importância de se criar sinergias para apoiar a gestão desses conhecimentos oriundos dessas práticas emergentes no seio da companhia.

\subsection{Cultura Organizacional}

A organização está completamente voltada para o conhecimento e, por isso, seus sócios percebem a necessidade urgente de implementar a GC. Diversas práticas de compartilhamento de conhecimento ocorrem - a exemplo de reuniões diárias de alinhamento de projetos, eventos de compartilhamento, dicas em e-mails e em ferramentas de chat de maneira informal, porém o Squad reconheceu a necessidade de estruturá-las para se ter constância e expandi-las em paralelo com outras iniciativas, facilitar o armazenamento e a sistematização desses conhecimentos para posterior recuperação e aplicação, o que acelera a qualidade das entregas.

Uma característica da cultura que reforça a oportunidade para o DP foi a participação da equipe de GC em eventos externos para capturar novos conhecimentos. $\mathrm{O}$ trabalho colaborativo e em equipe também mostrou-se muito presente, sendo uma característica não só do Squad de GC, mas de toda a companhia, em que as pessoas voluntariamente se dispõem a colaborar e dividir tarefas para dar agilidade aos projetos. Além disso, o departamento de Recursos Humanos fomenta a participação das pessoas nas mais diversas iniciativas da organização, comunicando e estimulando ações de engajamento para reforçar o papel da GC na empresa. Por outro lado, o fato de o projeto de implementação estar restrito a um grupo pequeno da organização dificultou a participação de todos de forma direta ao longo de todo o processo de implementação, o que deixou alguns colaboradores receosos da efetividade das práticas implementadas no médio e longo prazo, além da falta de compreensão sobre o tema da GC e sua interface com treinamento e desenvolvimento de pessoas.

Nesse contexto, o Design Participativo pode se mostrar importante para estimular as pessoas a colaborarem dentro dos mais diversos contextos existentes nas PMEs, aprimorando iniciativas porventura já existentes, mas sem estrutura, regularidade e consistência. No contexto estudado, uma iniciativa interessante poderia ser a prática de lições aprendidas de projetos anteriores em formato de workshops para trocar artefatos, conhecimentos e ideias que contribuam para a velocidade das rotinas em projetos futuros, reduzindo o retrabalho e melhorando a gestão do tempo nos projetos.

\section{Considerações finais}

Esse estudo aponta para a relevância do DP em apresentar algumas oportunidades para a implementação da GC em PMEs, por valorizar contextos que ajudam a enfrentar os fatores críticos de sucesso a iniciativas de GC em PMEs. Como síntese das oportunidades podemos destacar, para a 'estratégia', a participação de toda a alta direção na conformação das expectativas e diretrizes de um projeto de GC em PMEs, na medida em que os proprietários detêm a maior parte do conhecimento do negócio e eles são os primeiros a serem sensibilizados em qualquer projeto implementação. Além disso, todos membros da companhia precisam tomar conhecimento e participar das principais etapas 
do processo de implementação e das discussões sobre a GC, para facilitar a sinergia e comprometimento com as iniciativas.

No que tange à "liderança", os líderes devem dedicar tempo para o compartilhamento de seus conhecimentos e experiências aos liderados e incentivá-los a participar nas iniciativas de GC e nas discussões estratégicas, como demonstrado pela participação dos colaboradores em reuniões do CGE na empresa estudada. Por fim, quanto à "cultura organizacional", o DP poderia facilitar a compreensão dos membros da organização quanto à importância dos processos da GC, facilitar na definição de uma estratégia clara para a empresa - com o olhar representativo da maioria dos membros, independentemente do nível hierárquico - e estimular nos comportamentos dos líderes e liderados o compartilhamento do conhecimento, a integração, a sinergia e a confiança entre os membros da companhia na co-criação de iniciativas de GC que atendam às demandas práticas do dia a dia dos próprios colaboradores - usuários da GC - e na efetiva participação de práticas de GC institucionalizadas.

O DP apresenta-se como uma oportunidade para PMEs que queiram adotar a GC de forma deliberada, pois guarda profunda relação e imbricamento com os fatores críticos de sucesso vistos de forma conjunta ou separada, promovendo contextos favoráveis a ele. Pela análise dos dados, denota-se que a definição da estratégia depende do direcionamento da liderança, ao passo que a cultura depende dos outros dois fatores para auxiliar no alcance dos objetivos da organização. Desse modo, essa pesquisa apresenta uma contribuição original para o debate sobre a implementação da GC em PMEs, ao destacar a relevância do DP nesses contextos.

\section{Referências}

Anand, A.; Kant, R.; Patel, D. P. and Singh, M. D (2012). Knowledge Management Implementation: A Predictive Model Using an Analytical Hierarchical Process. Journal of the Knowledge Economy, v. 6, n. 1, p. 48-71.

Bahrami, M and Oktar, S. (2017). Identification and Ranking of Factors in Successful Implementation of Knowledge Management in Eghtesad Novin Bank: an Ahp Approach. IOSR Journal of Business and Management, 19(01), 58-63.

Bardin, L (1997). Análise de Conteúdo. Tradução Luís Antero Reto e Augusto Pinheiro. São Paulo: Edições 70.

Berthet, E. T. A., Barnaud, C., Girard, N., Labatut, J and Martin, G. (2016). How to foster agroecological innovations? A comparison of participatory design methods. Journal of Environmental Planning and Management, 59(2), 280-301.

Bratteteig, T and Wagner, I. (2014). Disentangling Participation: Power and Decision-Making in Participatory Design. Springer International Publishing.

Breiter, A. (2004). Requirements Development in Loosely Coupled Systems: Building a Knowledge Management System with Schools. Proceedings of the 37th Hawaii International Conference on System Sciences, 1-10.

Camargo, L. S. de A and Fazani, A. J. (2014). Explorando o Design Participativo como Prática de Desenvolvimento de Sistemas de Informação. InCID: Revista de Ciência Da Informação e Documentação, 5(1), 138.

Cepeda-Carrion, I., Martelo-Landroguez, S., Leal-Rodríguez, A. L and Leal-Millán, A. (2017). Critical processes of knowledge management: An approach toward the creation of 
customer value. European Research on Management and Business Economics, 23(1), 1-7.

Cerchione, R., Esposito, E and Spadaro, M. R. (2016). A literature review on knowledge management in SMEs. Knowledge Management Research and Practice, 14(2), 169-177.

Chang, T. H. and Wang, T. C (2009). Using the fuzzy multi-criteria decision making approach for measuring the possibility of successful knowledge management. Information Sciences, v. 179, n. 4, p. 355-370. Elsevier Inc.

Chen, X., Riedel, R., Bojko, M., Tawalbeh, M. and Egon, M. (2018). Knowledge Management as an Important Tool in Participatory Design. 541-548.

Davenport, T. H. and Prusak, L (1998). Conhecimento empresarial: como as organizações gerenciam o seu capital intelectual. 11. ed. Rio de Janeiro: Campus, 1998.

Dalkir, K (2017). Knowledge management in theory and practice. The MIT Press, 2017.

Deloitte. As PMEs que mais crescem no Brasil. 14ª Edição. 2019. Disponível em:

https://cutt.ly/cziLtQE. Acesso em: 12 ago 2020.

Delong, D.W. and Fahey, L (2000). Diagnosing cultural barriers to knowledge management. Academy of Management Executive, v. 14, n. 4, p. 113-127.

Despres, C. and Chauvel, D (2001). Knowledge horizons: The present and the promise of knowledge management. 2 ed. Butterworth-Heinemann: Boston, 2001.

Dorow, P. F. (2017). Compreensão do compartilhamento do conhecimento em atividades intensivas em conhecimento em organizações de diagnóstico por imagem. Tese (Doutorado) - Universidade Federal de Santa Catarina, Florianópolis, 2017.

Durst, S and Edvardsson, I. R. (2012). Knowledge management in SMEs: A literature review. Journal of Knowledge Management, 16(6), 879-903.

Durst, S. and Bruns, G. (2018), "Knowledge management in small and Medium-Sized enterprises", in The Palgrave Handbook of Knowledge Management, Palgrave Macmillan, Cham., pp. 495-514.

Govella, A. (2019). Collaborative Product Design. Help Any Team Build a Better Experience.

Hoffmann, M and Herrmann, T. (1999). A Design Process for Embedding Knowledge in Everyday Work Management. pp.296-305.

Kozinets, R. V (2014). Netnografia: realizando pesquisa etnográfica online. Porto Alegre: Penso, 2014.

Leedy, P. D and Ormrod, J. E. (2016). Practical Research: Planning and Design (11th ed.). Pearson.

Mahlamäki, K. (2013). The value of design information in collaborative design networks. International Conference on Engineering Design, ICED13, Conference Paper, 1-10.

Massaro, M., Handley, K., Bagnoli, C and Dumay, J. (2016). Knowledge management in small and medium enterprises: a structured literature review. In Journal of Knowledge Management (Vol. 20, Issue 2).

Miklosik, A and Zak, S. (2015). Framework for Effective Removal of Knowledge Management Implementation Barriers. Procedia Economics and Finance, 30(15), 513-521.

Mishra, N. (2019). Knowledge management practice for effective operations in SMEs. Production Planning and Control, 30(10-12), 795-798.

North, K. and Babakhanlou, R (2016). Knowledge Management Tools for SMES. Competitive Strategies for Small and Medium Enterprises, [s.l], p. 211-222. 
O'Dell, C. and Grayson, C. J. If only we knew what we know: identification and transfer of internal best practices. California Management Review, v. 40, n. 3, p. 154-174, 1998.

Ruggles, R (1998). The state of the notion: knowledge management in practice. California Management Review, v. 40, n. 3, p. 80-89.

Sakellariou, E.; Karantinou, K.; Goffin, K (2017). "Telling tales": Stories, metaphors and tacit knowledge at the fuzzy front-end of NPD. Creativity and Innovation Management, v. 26, n.4, p. 353-369.

Servin, G (2005). ABC of knowledge management. Disponível em: https://cutt.ly/dziKXql. Acesso em: 08 jul 2020.

Sebrae. Perfil das Microempresas e Empresas de Pequeno Porte 2018. Disponível em: https://cutt.ly/QziKxG0. Acesso em: 13 out. 2020.

Schuler, D. and Namioka, A. (2015). Participatory Design: Principles and Practices. Vol. 3, Issue 2. London: Taylor \& Francis.

Silverman, D. (2009). Interpretação de Dados Qualitativos. Métodos para Análise de Entrevistas, Textos e Interações. $3^{\text {a }}$ Ed. Porto Alegre: Bookman.

Straioto, R. G. T and Figueiredo, L. F. G. (2015). A co-criação sob a ótica da gestão de design: uma introdução aos níveis estratégico, tático e operacional do co-design. Fourth International Conference on Integration of Design, Engineering and Management for Innovation.

Teece, D. J., Pisano, G and Shuen, A. (1997). Dynamic capabilities and strategic management. Strategic Management Journal, 18(7), 509-533.

Valtolina, S., Barricelli, B. R and Dittrich, Y. (2012). Participatory knowledge-management design: A semiotic approach. Journal of Visual Languages and Computing, 23(2), 103115.

Van Der Bijl-brouwer, M.; Van Der Voort, M (2014). Establishing shared understanding of product use through collaboratively generating an explicit frame of reference. M. van der Bijl-Brouwer, Org. CoDesign, v. 10, n. 3-4, p. 171-190.

Wang, Y., Yu, S and Xu, T. (2017). A user requirement driven framework for collaborative design knowledge management. Advanced Engineering Informatics, 33, 16-28.

Wee, J. C. N and Chua, A. Y. K. (2013). The peculiarities of knowledge management processes in SMEs : the case of Singapore. Journal of Knowledge Management, 17(6), 958-972.

Wiig, K. M (1993). Knowledge management foundations: Thinking about thinking: How people and organizations create, represent, and use knowledge. Arlington, TX:Schema Press.

Wong, K.Y. (2005). Critical Success Factors for Implementing Knowledge Management in Small and Medium Enterprises. Industrial Management and Data Systems, 105(3), 261 279.

Wong, K. Y and Aspinwall, E. (2004). Characterizing knowledge management in the small business environment. Journal of Knowledge Management, 8(3), 44-61.

Wong, K. Y and Aspinwall, E. (2005). An empirical study of the important factors for knowledge-management adoption in the SME sector. Journal of Knowledge Management, 9(3), 64-82.

Yang, Y. O.; Yeh, J. and Lee, T (2010). The Critical Success Factors for Knowledge Management Adoption A Review Study. 3rd International Symposium on Knowledge Acquisition and Modeling, p. 445-448.

Yu, T. F. L (2001). Toward a capabilities perspective of the small firm. International Journal of Management Reviews, v. 3, n. 3, p. 185-197. 